\title{
Labour admission test: a screening test for foetal distress in labour
}

\author{
Mohd Rasheed $^{1 *}$, Ajay K. Srivastava ${ }^{2}$
}

\author{
${ }^{1}$ Department of Obstetrics and Gynaecology, AIIMS, New Delhi, India \\ ${ }^{2}$ Department of Obstetrics and Gynaecology, Command Hospital (EC), Alipore Road, Kolkata 700027, West Bengal, \\ India
}

Received: 03 December 2016

Accepted: 28 December 2016

*Correspondence:

Dr. Mohd Rasheed,

E-mail: rashiddr81077@gmail.com

Copyright: (c) the author(s), publisher and licensee Medip Academy. This is an open-access article distributed under the terms of the Creative Commons Attribution Non-Commercial License, which permits unrestricted non-commercial use, distribution, and reproduction in any medium, provided the original work is properly cited.

\begin{abstract}
Background: Labour is a very short period in the life of a foetus but poses the maximum threat. Improvements in medical technology have made it possible to monitor fetal well-being during labour. The introduction of labour admission test has proven to be of benefit in identifying patients at risk for developing distress and implementing timely intervention.

Methods: The present study was undertaken to evaluate the same. This was a prospective study done on 500 patients above 37 weeks of gestation in cephalic presentation. EFM was done using oxford sonicaid 8002 CTG machine and Gem premier 3000 (model no 5700) arterial blood gas analyzer machine was used for determining the $\mathrm{pH}$ of fetal blood.

Results: Statistical analysis was done using SPSS version 15.0 statistical analysis software. p value $<0.001$ was considered highly significant. Results revealed that a total of $105(80.77 \%)$ babies had fetal distress in category II and III out of 135 , whereas only $30(8.11 \%)$ were distressed in category I out of 370 . Test had a sensitivity of $77.7 \%$ and specificity of $93.15 \%$ with a positive predictive value of $80.7 \%$ and a negative predictive value of $91.89 \%$.

Conclusions: It has become evident that combined use of CTG with cord blood $\mathrm{pH}$ is more accurate way of predicting and diagnosing birth asphyxia.
\end{abstract}

Keywords: Cardiotocograph, Fetal distress, Fetal hypoxia, Labour admission test, NICU

\section{INTRODUCTION}

It is well established that labour poses physiological stress to all foetuses during the transition from the intrauterine to the extrauterine environment. ${ }^{1}$ This led to introduction of various fetal surveillance measures which would detect any fetal distress and lead to timely intervention. One such measure to this end is "labour admission test".

This test was introduced by Ingemarsson and comprises of a CTG trace of 20-30 minutes duration carried out on admission to the labour room. The aim of the test is to assess fetal well-being at the onset of labor and identify those fetuses that may be already hypoxic or may not withstand the stress of uterine contractions which can expose them to hypoxia in labour. ${ }^{2}$

Electronic monitoring of FHR which is a routine practice in developed countries but has limited routine use in developing countries due to economic considerations thereby requiring selection of fetuses for continuous monitoring. ${ }^{3}$

This study was undertaken with the purpose of evaluating the efficacy of labour admission test in identifying, early in labor, the fetuses at high risk of developing intrapartum distress. The confirmation of the same was done by cord blood $\mathrm{pH}$ values. 


\section{METHODS}

This was a prospective cohort study of patients in $1^{\text {st }}$ stage of labour admitted to labour room at a Tertiary care hospital and was carried over a period of one year from 1st November 2009 to $31^{\text {st }}$ October 2010.

A total of 500 cases at more than 37 weeks period of gestation with cephalic presentation, in $1^{\text {st }}$ stage of labour were included. Pregnant women who were preterm, having multiple gestation, in $2^{\text {nd }}$ stage of labour, hypertension, diabetes mellitus, APLA syndrome, Intra Uterine Growth Restriction, oligohydramnios, polyhydramnios, congenital malformation detected on ultrasonography, any concurrent medical illness were excluded. A written informed consent was taken after explaining the procedure in detail. The study protocol was approved by the institutional ethics committee.

On admission to labour room, a detailed history, general physical, per abdominal and vaginal examination was done. Thereafter, patients were subjected to admission test for $20 \mathrm{~min}$. The EFM was carried out using Oxford Sonicaid 8002 cardiotocography (CTG) machine where one probe was meant to pick up continuous tracing of FHR and other for uterine contractions.

The paper speed was set at $1 \mathrm{~cm}$ per minute. Five components of the tracing were identified namely Baseline heart rate, Beat to Beat variability, Accelerations, Decelerations and Sinusoidal pattern.

The results of admission test were categorized into,

- Category I: FHR tracings are normal

- Category II: FHR tracings are indeterminate

- Category III: FHR tracing are abnormal (as per American College of Obstetrics and Gynaecologists (ACOG) Practice bulletin. No 106, guidelines for interpretation of CTG tracing. ${ }^{4}$

Patients with category I admission test were monitored by intermittent auscultation for $1 \mathrm{~min}$, every $30 \mathrm{~min}$ during $1^{\text {st }}$ stage of labour and every $5 \mathrm{~min}$ during $2^{\text {nd }}$ stage of labour.

Those having category II tracing were monitored by continuous CTG monitoring or intensive auscultatory methods.

Those with category III trace were taken as foetal distress.

Delivery conducted was either by normal vaginal route, instrumental vaginal delivery or by caesarean section depending upon the foetal heart rate tracings and their interpretations as per the case. Colour of liquor was noted at the time of delivery. A sample of umbilical cord blood was taken for $\mathrm{pH}$ analysis.
All new born babies were assessed after the delivery and at 1 and 5 minute Apgar score noted. Babies having low Apgar score or any other complication were shown to paediatrician immediately. The neonatal outcome was also recorded including the requirement of NICU admission.

Gem Premier 3000 (model no 5700) Arterial Blood Gas analyzer machine was used for determining the $\mathrm{pH}$ of foetal blood and results were compared with CTG trace findings.

The various EFM Patterns obtained were compared with the neonatal status at birth using the parameters already mentioned. The false positives and false negatives if any were calculated

For the present analysis, the outcome variables were taken as:

- Apgar Score at 1 min $(>7$ or $<7)$

- Apgar Score at 5 min $(>9$ or $<9)$

- MSL (Nil, Thin or Thick)

- Mode of delivery [Vaginal, Instrumental (vacuum or forceps) or LSCS]

- Cord blood pH (<7.25 or $\geq 7.25)$

- NICU Admission

\section{Statistical analysis}

Data analysis and statistical verification was done by Nonparametric chi-square test using SPSS Version 15.0 statistical analysis software. Statistical significance was calculated between different categories for different parameters too. A $p$ value of of $<0.05$ was considered to be statistically significant.

\section{RESULTS}

The present study had majority of women in the age group of 25-30 years (43.6\%) and $53.6 \%$ were multigravida. Normal FHR tracings were observed in $74 \%$ women, whereas $17.8 \%$ had indeterminate and $8.2 \%$ had abnormal results (Table 1).

Results for different parameters in all the three categories of CTG are described in Table 2 .

The incidence of LSCS delivery, low Apgar at 1 and 5 min and NICU admission was significantly higher in category II and III as compared to category I. As compared to category I, the incidence of LSCS delivery, low Apgar at 1 and $5 \mathrm{~min}$ and NICU admission was significantly higher among subjects with category III findings. No significant difference between category II and category III was observed for thick MSL and low Apgar at $5 \mathrm{~min}$, however, for other outcomes, the proportion of adverse outcome was significantly higher among subjects with category III findings as compared to those with category II findings (Table 3 ). 
Table 1: Distribution of subjects with different categories of CTG Findings according to age and parity.

\begin{tabular}{|c|c|c|c|c|c|c|c|c|}
\hline \multirow[t]{2}{*}{ Age (years) } & \multicolumn{2}{|c|}{ Category I } & \multicolumn{2}{|c|}{ Category II } & \multicolumn{2}{|c|}{ Category III } & \multicolumn{2}{|c|}{ Total $(n=500)$} \\
\hline & No & $\%$ & No & $\%$ & No & $\%$ & No & $\%$ \\
\hline $20-25$ & 137 & 37 & 39 & 43.8 & 19 & 46.3 & 195 & 39 \\
\hline $25-30$ & 169 & 45.7 & 33 & 37.1 & 16 & 39.0 & 218 & 43.6 \\
\hline$>30$ & 64 & 17.3 & 17 & 19.1 & 6 & 14.6 & 87 & 17.4 \\
\hline Total & 370 & 74.0 & 89 & 17.8 & 41 & 8.2 & & \\
\hline Parity & & & & & & & & \\
\hline Primi & 171 & 46.2 & 39 & & 22 & 53.7 & 232 & 46.4 \\
\hline Multi & 199 & 53.8 & 50 & & 19 & 46.3 & 268 & 53.6 \\
\hline
\end{tabular}

Table 2: Distribution of subjects with different categories of CTG findings according to Apgar score, meconium findings, mode of delivery, $\mathrm{pH}$ and NICU admissions.

\begin{tabular}{|c|c|c|c|c|c|c|}
\hline \multirow{2}{*}{ Apgar score at $1 \mathrm{~min}$} & \multicolumn{2}{|c|}{ Category I (n=370) } & \multicolumn{2}{|c|}{ Category II (n=89) } & \multicolumn{2}{|c|}{ Category III (n=41) } \\
\hline & No. & $\%$ & No. & $\%$ & No. & $\%$ \\
\hline$<7$ & 20 & 5.4 & 69 & 77.5 & 21 & 51.2 \\
\hline$\geq 7$ & 350 & 94.6 & 20 & 22.5 & 20 & 48.8 \\
\hline \multicolumn{7}{|c|}{$\chi^{2}=239.694,(\mathrm{df}=2) ; \mathrm{p}<0.001$} \\
\hline \multicolumn{7}{|c|}{ Apgar score at $5 \mathrm{~min}$} \\
\hline$<9$ & 0 & 0.0 & 25 & 28.1 & 10 & 24.4 \\
\hline$\geq 9$ & 370 & 100.0 & 64 & 71.9 & 31 & 75.6 \\
\hline \multicolumn{7}{|c|}{$\chi^{2}=107.73,(\mathrm{df}=2) ; \mathrm{p}<0.001$} \\
\hline \multicolumn{7}{|c|}{$\mathrm{pH}$} \\
\hline$\leq 7.25$ & 30 & 8.1 & 69 & 77.5 & 36 & 87.8 \\
\hline$>7.25$ & 340 & 91.9 & 20 & 22.5 & 5 & 12.2 \\
\hline \multicolumn{7}{|c|}{$\chi^{2}=259.19,(\mathrm{df}=2) ; \mathrm{p}<0.001$} \\
\hline \multicolumn{7}{|c|}{ Mode of delivery } \\
\hline FTND & 335 & 90.5 & 31 & 34.8 & 0 & 0.0 \\
\hline Instrumental & 0 & 0.0 & 19 & 21.3 & 3 & 7.3 \\
\hline LSCS & 35 & 9.5 & 39 & 43.8 & 38 & 92.7 \\
\hline \multicolumn{7}{|c|}{$\chi^{2}=274.589,(\mathrm{df}=2) ; \mathrm{p}<0.001$} \\
\hline \multicolumn{7}{|c|}{ Meconium findings } \\
\hline Normal & 304 & 82.2 & 66 & 74.2 & 30 & 73.2 \\
\hline Thin & 36 & 9.7 & 5 & 5.6 & 2 & 4.9 \\
\hline thick & 30 & 8.1 & 18 & 20.2 & 9 & 21.9 \\
\hline \multicolumn{7}{|c|}{$\chi^{2}=161.55,(\mathrm{df}=2) ; \mathrm{p}<0.001$} \\
\hline \multicolumn{7}{|c|}{ NICU admissions } \\
\hline No & 370 & 100 & 69 & 77.5 & 21 & 51.2 \\
\hline yes & 0 & 0.0 & 20 & 22.5 & 20 & 48.8 \\
\hline
\end{tabular}

Table 3: Between category comparison for different outcomes.

\begin{tabular}{|llllllll|}
\hline \multirow{2}{*}{ SN } & Outcome & \multicolumn{2}{c|}{ Category I vs Category II } & \multicolumn{2}{c|}{ Category I vs Category III } & \multicolumn{2}{c|}{ Category II vs Category III } \\
\hline 1. & LSCS delivery & $\boldsymbol{\chi}^{\mathbf{2}}$ & $\mathbf{p}$ & $\boldsymbol{\chi}^{\mathbf{2}}$ & $\mathbf{p}$ & $\boldsymbol{\chi}^{\mathbf{2}}$ & $\mathbf{P}$ \\
\hline 2. & Apgar $<7$ at 1 min & 109.92 & $<0.001$ & 175.02 & $<0.001$ & 27.753 & $<0.001$ \\
\hline 3. & Apgar $<9$ at 5 min & 109.92 & $<0.001$ & 86.266 & $<0.001$ & 9.120 & 0.003 \\
\hline 4. & NICU admission & 86.934 & $<0.001$ & 92.494 & $<0.001$ & 0.195 & 0.659 \\
\hline
\end{tabular}


A total of $105(80.77 \%)$ babies had fetal distress in category II and III whereas only 30 (8.11\%) were distressed in category I (Table 4).

Table 4: Admission test interpretation viz-a-viz ability to identify fetal distress.

\begin{tabular}{|llll|}
\hline & $\begin{array}{l}\text { Fetal } \\
\text { distress }\end{array}$ & $\begin{array}{l}\text { No Fetal } \\
\text { distress }\end{array}$ & \\
\hline Cat II+ Cat III* & $105(80.77 \%)$ & $25(19.23 \%)$ & 130 \\
\hline Cat I & $30(8.11 \%)$ & $340(91.89 \%)$ & 370 \\
\hline Total & 135 & 365 & 500 \\
\hline
\end{tabular}

*For analysis purposes, Cat II and Cat III have been taken together.

The sensitivity and specificity of the admission test is given in (Table 5).

Table 5: Sensitivity and specificity of test.

\begin{tabular}{|ll|} 
Parameter & Percentage \\
\hline Sensitivity & 77.7 \\
\hline Specificity & 93.15 \\
\hline PPV & 80.7 \\
\hline NPV & 91.89 \\
\hline
\end{tabular}

\section{DISCUSSION}

Even though labour and delivery is regarded as a normal physiological process, the intrapartum complications can arise very quickly and unexpectedly in both high and low risk pregnancy. ${ }^{5}$

LAT helps to identify those foetuses that may be already be hypoxic or may not withstand the stress of uterine contractions which can expose them to hypoxia in labour. ${ }^{6}$

So, LAT can be used as a screening tool in early labour to identify unsuspected cases of fetal jeopardy that may benefit with continuous electronic fetal heart monitoring during labour. ${ }^{6}$

The present study showed a significant difference in Apgar score at 1 min among different categories of CTG findings (Table 2). Similar observations were made for Apgar score at 5 min. Similar differences have been shown in studies by Gauge SM and Pearson J. ${ }^{7,8}$

In present study it is noted that there is no difference between the colour of liquor in the three categories (Table 2). These same findings have also been reported in studies by Gurung G, Abramovici $\mathrm{H}$ and Miller FC. ${ }^{9-11}$

Majority of category II and category III subjects in this study had a $\mathrm{pH}$ of $<7.25$ whereas majority of subjects in category I had $\mathrm{pH}$ values $\geq 7.25$. Statistically, there was a significant association between CTG category and $\mathrm{pH}$ category (Table 2). In a similar study by Bahiah AS, a scalp blood $\mathrm{pH}$ of less than 7.20 or base excess-10 demonstrates a significant fetal metabolic acidosis indicating fetal hypoxia. ${ }^{12}$

In category I in this study, most of the subjects (90.5\%) had full term normal delivery and remaining 35 (9.5\%) had cesarean delivery. In category II, maximum subjects had cesarean delivery $(43.8 \%)$ followed by those having full term normal delivery $(34.8 \%)$ and those having instrumental delivery $(21.3 \%)$. Among category III subjects maximum had LSCS delivery $(92.7 \%)$ followed by those having instrumented delivery $(7.3 \%)$, none of the subjects with category III CTG findings had full term normal delivery. A distinct and statistically significant difference in mode of delivery among subjects with different category of CTG findings was observed $(p<0.001)$ (Table 2). These results are in concordance with studies by Gurung G, Hegde A, Kulkarni AA and Bhat RA. ${ }^{9,13-15}$

In the current study none of the neonates born to women with category I CTG findings required NICU admission. However, a total of 20 (22.5\%) neonates born to category II CTG finding mothers and a total of 20 (48.8\%) neonates born to category III CTG finding mothers required NICU admission (Table 2). This difference was found to be statistically significant $(\mathrm{p}<0.001)$. The reason for the NICU admissions in present study was hypoxia and neonatal sepsis. Similar results have been seen in studies by Gurung $\mathrm{G}$ and Perveen S. ${ }^{9,16}$

The predictive ability of the admission test was evaluated to identify the foetuses at high risk for developing intrapartum distress. As evident in Table 5, the sensitivity or true positive rate of the admission test is seen to be low $(77.7 \%)$ with high specificity $(93.15 \%)$ meaning thereby that the pickup rate of the test is relatively less. This suggests that a positive result may helpfully identify the patients albeit at the risk of missing some patients where intervention is otherwise warranted. Further the negative predictive value is very high $(91.8 \%)$ as compared to positive predictive value of $80.7 \%$; it means that a negative test in stills more confidence in the clinician's mind as compared to a positive test result. These results suggest that admission test can be used as an important non-invasive method to diagnose foetal compromise present at the time of admission in early labour.

\section{CONCLUSION}

Results of admission test with perinatal outcome and mode of delivery as observed in the present study have shown that it is a good, economical, non invasive, readily available screening test and can predict fetal well being during the next few hours of labor. Also the combined use of CTG with cord blood $\mathrm{pH}$ makes it more accurate way of predicting and diagnosing birth asphyxia. This test would prove to be a useful screening tool for detecting early fetal distress in developing countries with limited resource settings. However, the major risk 
associated with this is a false positive test that may result in unnecessary surgical intervention.

Funding: No funding sources Conflict of interest: None declared

Ethical approval: The study was approved by the Institutional Ethics Committee

\section{REFERENCES}

1. Nelson MD, Alan Leviton. How much of neonatal encephalopathy is due to birth asphyxia? Am J Dis Child. 1991;145(11):1325-31.

2. Ingemarsson I, Arulkumaran S, Ingemarsson E, Tambyraja RL, Ratnam SS. Admission test: A screening test for fetal distress in labor. Obstet Gynecol. 1986;68(6):800-6.

3. Rahman H, Renjhen P, Dutta S, Kar S. Admission cardiotocography; its role in predicting fetal outcome in high risk obstetric patients. Australas Med J. 2012;5(10);522-7.

4. American College of Obstetricians and Gynaecologists. Practice Bulletin No 106, July; 2009.

5. Nikita V, Kumare B. Labour Admission Test (LAT) as a Predictor of Intrapartum Fetal Distress. Panacea Journal of Medical Sciences. 2016;6(1):26-30.

6. Talaulikar VS, Arulkumaran S. Labour admission test. Intl J Infer Fetal Med. 2011;2(3):89-95.

7. Gauge SM, Handerson C. CTG made easy. Churchil Livingstone, Singapore. 1997:9-13.

8. Pearson J, Raees G. Technique of cesarean section. In: Charlmers I, Enkin M , Keirs MJNC (edi).
Effective care in pregnancy and child birth: childbirth Oxford University Press; 1989:1234-69.

9. Gurung G, Rana A, Giri K. Detection of intrapartum fetal hypoxia using admission test (AT) N. J. Obstet. Gynaecol. 2006;1(2):10-3.

10. Abramovici H, Brandes JM, Fuchs K. Meconium during delivery: A sign of compensated distress. Am J Obstet Gynecol. 1974;118-25.

11. Miller FC, Sacks DA, Yeh SY, Paul RH, Schifrin BS, Martin CB, et al. Significance of meconium during labor. Am J Obstet Gynecol. 1975;122(5):573-80.

12. Bahiah AS, Murphy JF, Sharida HE. Fetal Distress in Labor and Caesarian Section Rate. Bahrain Medical Bulletin. 2010;32(2).

13. Hegde A, Kore S, Srikrishna S, Ambiye VR, Vaidya PR: Admission test : Screening test for prediction of fetal outcome in labour. $\mathbf{J}$ of Obst. and Gyn. of India. 2001;51:40.

14. Kulkarni AA, Shroti AN. Admission Test: A predictive test for distress in high risk labor. J Obstet Gynecol Res. 1998;24(4):255-9.

15. Bhat RA. Labour admission test: A screening tool. Obs and Gynae Today. 2006;9(6):328-31.

16. Perveen S, Hashmi H. Effectiveness of admission test. JDUHS. 2007;1(1):20-5.

Cite this article as: Mohd R, Srivastava AK. Labour admission test: a screening test for foetal distress in labour. Int J Reprod Contracept Obstet Gynecol 2017;6:452-6. 